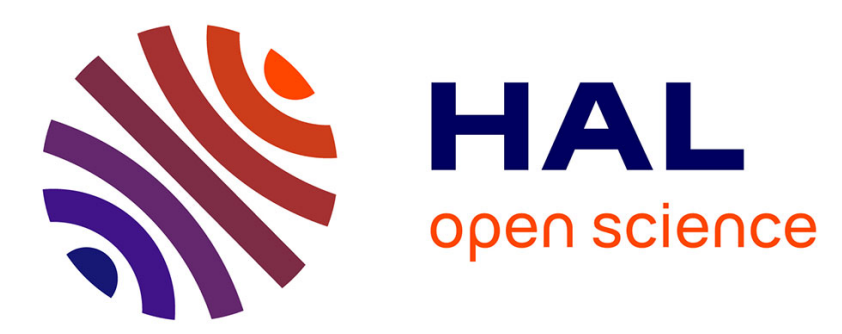

\title{
Analyse expérimentale des phénomènes de conversion liés à l'interaction d'une onde de Rayleigh avec une rainure de forme rectangulaire
}

\author{
E. Baron, M. de Billy, G. Quentin
}

\section{- To cite this version:}

E. Baron, M. de Billy, G. Quentin. Analyse expérimentale des phénomènes de conversion liés à l'interaction d'une onde de Rayleigh avec une rainure de forme rectangulaire. Revue de Physique Appliquée, 1985, 20 (6), pp.369-375. 10.1051/rphysap:01985002006036900 . jpa-00245346

HAL Id: jpa-00245346

https://hal.science/jpa-00245346

Submitted on 1 Jan 1985

HAL is a multi-disciplinary open access archive for the deposit and dissemination of scientific research documents, whether they are published or not. The documents may come from teaching and research institutions in France or abroad, or from public or private research centers.
L'archive ouverte pluridisciplinaire HAL, est destinée au dépôt et à la diffusion de documents scientifiques de niveau recherche, publiés ou non, émanant des établissements d'enseignement et de recherche français ou étrangers, des laboratoires publics ou privés. 


\title{
Analyse expérimentale des phénomènes de conversion liés à l'interaction d'une onde de Rayleigh avec une rainure de forme rectangulaire $\left(^{+}\right)$
}

\author{
E. Baron (*), M. de Billy et G. Quentin \\ Groupe de Physique des Solides de l'Ecole Normale Supérieure, Université Paris 7, Tour 23, \\ 2, place Jussieu, 75251 Paris Cedex 05, France
}

(Reçu le 4 octobre 1984, révisé le 15 février 1985, accepté le 11 mars 1985)

\begin{abstract}
Résumé. - Nous nous proposons d'étudier les propriétés directionnelles des ondes de volume engendrées par l'interaction d'une onde de surface avec une rainure de forme rectangulaire usinée au centre de la face plane d'un demi-cylindre. En nous limitant au problème à deux dimensions (la rainure est supposée infinie suivant l'axe du cylindre), nous avons mesuré les diagrammes angulaires $S_{j}(\theta)(\theta$ est l'angle sagittal) représentatifs de l'intensité normalisée emportée par chaque type d'onde de volume. Les résultats expérimentaux sont discutés et comparés avec les calculs théoriques basés sur la technique des petites perturbations développée par Parekh et Tuan [ $J$. Appl. Phys. 48 (1977) 994-1003].
\end{abstract}

\begin{abstract}
In this paper, we study the surface wave to bulk wave conversion of a Rayleigh wave incident on a rectangular groove engraved on a duralumin sample. The depth $h$ of the groove verify the condition $\varepsilon=h / \lambda_{\mathrm{R}} \ll 1$ to be in agreement with the theoretical assumption ( $\lambda_{R}$ is the wavelength of the Rayleigh wave in the medium). The angular variations of the incident power converted into bulk waves are measured versus the sagittal angle $\theta$. The experimental results are discussed and compared with the theoretical model based on the perturbation technique developed by Parekh and Tuan [J. Appl. Phys. 48 (1977) 994-1003].
\end{abstract}

\section{Introduction.}

Le contrôle non destructif des matériaux (NDE) ainsi que le traitement du signal sont deux domaines qui ont motivé jusqu'à présent de nombreuses études concernant en particulier l'interaction d'ondes acoustiques de surface avec des discontinuités superficielles.

Ces ondes qui se propagent à la surface des milieux élastiques homogènes et dont l'amplitude décroît rapidement avec la profondeur sont mathématiquement bien définies et relativement faciles à générer expérimentalement. Ce type d'onde acoustique est utilisé pour rechercher et localiser des défauts de petite taille, de dimensions, en général, inférieures ou égales à la longueur d'onde. On peut observer alors des phénomènes de diffraction, de réflexion et de diffusion de l'onde incidente. Ce dernier effet est le plus étudié

$\left({ }^{+}\right)$Cette étude a été soutenue par la DRET (Contrat no 83-070).

(*) ETCA, 16 bis, avenue Prieur-de-la-Côte-d'Or, 94114 Arcueil Cedex, France. et le plus révélateur aussi, car il permet de résoudre le problème inverse : caractériser un défaut connaissant la figure de diffusion qu'il engendre.

Parfois, des inhomogénéités artificielles (telles que des rainures, des marches... simples ou en réseaux périodiques) sont gravées en surface pour des applications pratiques. Lorsqu'une onde de surface rencontre ce type de défauts, elle est partiellement convertie en ondes de volume. Dans le cas des dispositifs à ondes de surface (SAW), cette génération d'onde de volume représente une perte d'énergie non souhaitée. Par contre, il faut souligner le développement d'appareils (grating scanners) à balayage pour lesquels la génération d'ondes de volume est souhaitée et doit être favorisée [1]. De ces brèves considérations, il ressort que les phénomènes de conversion d'ondes de surface en ondes de volume est un problème important qui mérite une attention toute particulière.

Les différents phénomènes (réflexion, transmission et conversion de mode) qui peuvent se produire lorsqu'une onde de surface interagit avec une rainure superficielle ont été étudiés théoriquement. Tuan et 
Li [2] ainsi que Simons [3] ont étudié la réflexion d'une onde de Rayleigh par une rainure de forme rectangulaire. Simons a généralisé l'analyse précédente au cas d'un réseau périodique de rainures ou de marches.

En ce qui concerne la conversion d'une onde de surface en ondes de volume, Tuan [4] et Parekh [5] ont développé une approche théorique distinguant les rainures à fond plat des autres profils.

Le problème théorique a été résolu par la technique des petites perturbations au premier ordre [6-8]. Ronnekleiev et Souquet $[9,10]$ ont utilisé la même approche pour étudier le cas d'un ensemble infini de rainures. Ces recherches ont montré que, quel que soit le type de défaut, les ondes de volume sont engendrées et que la distribution angulaire de l'énergie portée par ces ondes dépend très fortement de la largeur de la fente et non de son profil. Les propriétés directionnelles de ces ondes de volume peuvent être décrites par une fonction sans dimension $S_{j}(\theta)$ où $j=l$ pour une onde de volume longitudinale et $j=l$ pour une onde de volume transversale. Cette fonction représente le diagramme angulaire de l'intensité acoustique normalisée transportée par les ondes de volumes dans la direction $\theta$ ( $\theta$ est l'angle sagittal et représente la variable).

Nous avons limité nos mesures au cas de rainures de forme rectangulaire tracées sur la face plane d'un demicylindre de duraluminium et selon l'axe du cylindre. Les expériences ont été conduites à différentes fréquences de façon à observer l'évolution des diagrammes $S_{j}(\theta)$ en fonction du rapport $\varepsilon=h / \lambda_{\mathrm{R}}$ où $h$ désigne la profondeur de la rainure et $\lambda_{R}$ la longueur d'onde associée à l'onde de Rayleigh incidente.

\section{Rappels théoriques.}

Dans cette partie, nous résumons la formulation théorique proposée par Tuan pour calculer l'intensité acoustique transportée par les ondes de volume engendrées lors de l'interaction d'une onde de Rayleigh avec une rainure superficielle de profil quelconque mais supposée infinie dans la direction perpendiculaire à la direction de propagation $\mathrm{O} X$ de l'onde incidente

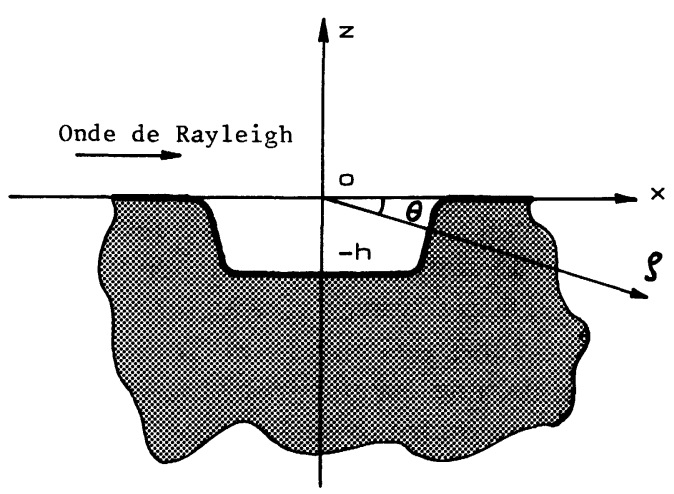

Fig. 1. - Géométrie du problème. (voir Fig. 1). Evaluer cette intensité revient à calculer le vecteur de Poynting donné par la relation :

$$
\hat{\mathbf{P}}=\frac{1}{2} \operatorname{Re}\left(i \omega \hat{\mathbf{u}} T^{*}\right)
$$

dans laquelle û représente le vecteur déplacement et $T^{*}$ le complexe conjugué du tenseur des contraintes. Après calculs, on peut montrer que le vecteur $\hat{\mathbf{P}}$ est purement radial et peut s'écrire :

$$
\hat{\mathbf{P}}=\hat{\boldsymbol{\rho}}\left[S_{\mathrm{vl}}(\theta)+S_{\mathrm{vt}}(\theta)\right]
$$

avec

$$
S_{\mathrm{vl}}(\theta)=\frac{\omega k_{1}^{3}(\lambda+2 \mu)}{2}\left|\varepsilon \varphi_{1}(\rho, \theta)\right|^{2}
$$

et

$$
S_{\mathrm{vt}}(\theta)=\frac{\omega k_{\mathrm{t}}^{3} \mu}{2}\left|\varepsilon \psi_{1}(\rho, \theta)\right|^{2}
$$

$\hat{\boldsymbol{\rho}}$ est le vecteur unitaire dans la direction de propagation, $\lambda$ et $\mu$ sont les coefficients de Lamé,

$$
k_{1}=\frac{\omega}{C_{1}}, \quad k_{\mathrm{t}}=\frac{\omega}{C_{\mathrm{t}}} \quad \text { et } \quad k_{\mathrm{R}}=\frac{\omega}{C_{\mathrm{R}}}
$$

$\omega$ est la pulsation, $C_{1}, C_{\mathrm{t}}$ et $C_{\mathrm{R}}$ sont les célérités des différents types d'ondes.

$\varepsilon$ est un nombre sans dimension, très petit devant 1 $(\varepsilon \ll 1)$.

$\varphi_{1}$ et $\psi_{1}$ représentent les premiers termes du développement en série des champs rayonnés dans le solide.

Nous allons évaluer ces potentiels dans le cas particulier d'un profil rectangulaire.

L'échantillon qui se situe dans le demi-espace $(Z<0)$ est supposé élastique, isotrope et homogène. La rainure de largeur $2 a$ présente une section droite constante dans la direction $\mathrm{O} Y$ et peut être décrite par l'équation :

$$
S(X, Z)=Z-\varepsilon \lambda_{\mathrm{R}} f(X)=0
$$

où $f(X)$ est une fonction qui caractérise la forme de la rainure avec $f(0)=-1$ pour tous les profils. (Pour un profil rectangulaire, $f(X)$ est définie sur la Fig. 2.) Cette dernière propriété impose que la profondeur $h=\lambda_{\mathrm{R}} \varepsilon f(\mathrm{o})=-\lambda_{\mathrm{R}} \varepsilon$ soit très petite devant la longueur d'onde de Rayleigh $\lambda_{R}$. Cette inégalité étant satisfaite, Tuan [4] a montré que les solutions au premier ordre des potentiels $\varepsilon \varphi_{1}$ et $\varepsilon \psi_{1}$ pouvaient s'écrire en coordonnées polaires $(\rho, \theta)$ et pour des valeurs élevées de $k_{1} \rho$ et $k_{\mathrm{t}} \rho$ (champ lointain) sous la forme suivante :

$$
\begin{aligned}
& \varepsilon \varphi_{1}(\rho, \theta)=\frac{\varepsilon}{\sqrt{2 \pi}}\left(\frac{1}{k_{1} \rho}\right)^{1 / 2} \exp \left[i\left(\frac{3 \pi}{4}+k_{1} \rho\right)\right] \varphi_{1}^{\prime}(\theta) \\
& \varepsilon \psi_{1}(\rho, \theta)=\frac{\varepsilon}{\sqrt{2 \pi}}\left(\frac{1}{k_{\mathrm{t}} \rho}\right)^{1 / 2} \exp \left[i\left(\frac{3 \pi}{4}+k_{\mathrm{t}} \rho\right)\right] \psi_{1}^{\prime}(\theta)
\end{aligned}
$$

[Geometry of the problem.] 


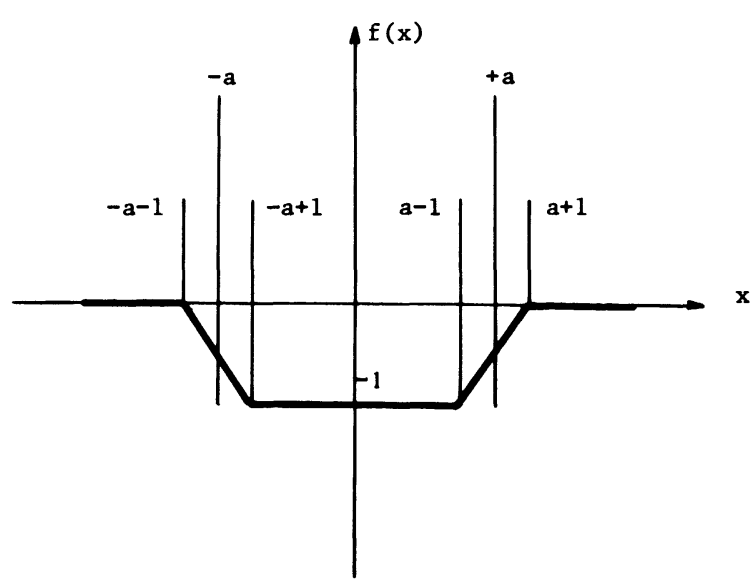

Fig. 2. - Définition du profil $f(x)$ :

$$
\begin{aligned}
& f(x)=0 \text { pour }|x| \geqslant a+l \\
& f(x)=-1 \text { pour }|x| \leqslant a-l \\
& f(x)=-\frac{1}{2 l}(x+a+l) \text { pour }-a-l \leqslant x \leqslant-a+l \\
& f(x)=\frac{1}{2 l}(x-a-l) \text { pour } a-l \leqslant x \leqslant a+l .
\end{aligned}
$$

[Definition of the profile $f(x)$.]

où

$$
\begin{aligned}
& \varphi_{1}^{\prime}(\theta)=-\frac{k_{1}}{F(k)}\left[2 k \xi_{\mathrm{t}} \frac{P(k)}{\mu}+\right. \\
& \left.+\left(2 k^{2}-k_{\mathrm{t}}^{2}\right) \frac{Q(k)}{\mu}\right] \sin \theta \\
& \psi_{1}^{\prime}(\theta)=-\frac{k_{\mathrm{t}}}{F(k)}\left[2 k \xi_{1} \frac{Q(k)}{\mu}+\right. \\
& \left.+\left(k_{\mathrm{t}}^{2}-2 k^{2}\right) \frac{P(k)}{\mu}\right] \sin \theta \\
& \xi_{1}=\left(k_{1}^{2}-k^{2}\right)^{1 / 2} \text { pour }|k|<k_{1}
\end{aligned}
$$

et

$$
\begin{gathered}
i\left(k^{2}-k_{1}^{2}\right)^{1 / 2} \text { pour }|k|>k_{1}, \\
\xi_{\mathrm{t}}=\left(k_{\mathrm{t}}^{2}-k^{2}\right)^{1 / 2} \text { pour }|k|<k_{\mathrm{t}}
\end{gathered}
$$

et

$$
i\left(k^{2}-k_{\mathrm{t}}^{2}\right)^{1 / 2} \text { pour }|k|>k_{\mathrm{t}},
$$

$F(k)=4 k^{2} \xi_{t} \xi_{1}+\left(k_{t}^{2}-2 k^{2}\right)^{2}$ étant l'équation de dispersion de Rayleigh.

Les fonctions $P(k)$ et $Q(k)$ sont données par les équations (10a) et (10b) de la référence [5]. Elles dépendent de la forme du profil par la fonction $\mathrm{H}(\mathrm{k})$. Dans le cas du profil étudié (rectangulaire), nous trouvons :

$$
\begin{aligned}
H(k)=-\frac{2}{l\left(k-k_{\mathrm{R}}\right)^{2}}[\sin ( & \left.\left.k-k_{\mathrm{R}}\right) a\right] \times \\
& \times\left[\sin \left(k-k_{\mathrm{R}}\right) l\right] .
\end{aligned}
$$

La quantité $l$ contrôle la pente de la rainure et a été choisie de manière à satisfaire la condition $l \ll a$ (cas d'une rainure rectangulaire). Connaissant les expressions des potentiels $\varepsilon \varphi_{1}$ et $\varepsilon \psi_{1}$, il est alors possible d'évaluer $S_{\mathrm{vl}}(\rho, \theta)$ et $S_{\mathrm{vt}}(\rho, \theta)$.

L'étude présente ne s'intéressant qu'aux propriétés directionnelles des ondes de volume engendrées dans le solide, il apparaît plus adapté, en accord avec Tuan et Parekh, de définir à partir des équations (3) et (4) deux fonctions $S_{1}(\theta)$ et $S_{\mathrm{v}}(\theta)$ représentatives des diagrammes angulaires de l'intensité normalisée suivant le type d'onde étudiée. Nous trouvons alors :

$$
\begin{aligned}
& S_{1}(\theta)=\frac{2 \rho k_{\mathrm{R}}^{4}}{\varepsilon^{2} \omega k_{1}^{2}} \cdot \frac{1}{(\lambda+2 \mu)} S_{1}(\rho, \theta) \\
& S_{\mathrm{t}}(\theta)=\frac{2 \rho k_{\mathrm{R}}^{4}}{\varepsilon^{2} \omega k_{\mathrm{t}}^{2}} \cdot \frac{1}{\mu} \cdot S_{\mathrm{t}}(\rho, \theta)
\end{aligned}
$$

soit en fonction des potentiels :

$$
\begin{aligned}
& S_{1}(\theta)=\left|k_{\mathrm{R}}^{2} \varphi_{1}(\rho, \theta)\right|^{2} k_{1} \rho \\
& S_{\mathrm{t}}(\theta)=\left|k_{\mathrm{R}}^{2} \psi_{1}(\rho, \theta)\right|^{2} k_{\mathrm{t}} \rho
\end{aligned}
$$

Sur la figure 3, nous avons reproduit quelques tracés théoriques typiques représentant les variations en fonction de l'angle sagittal $\theta$, des quantités :

$$
\begin{aligned}
& S_{1}^{\prime}(\theta)=\left[\frac{k_{\mathrm{t}}}{k_{\mathrm{R}}}\right]^{2} \frac{S_{\mathrm{l}}(\theta)}{2 W_{\mathrm{i}}} \\
& S_{\mathrm{t}}^{\prime}(\theta)=\left[\frac{k_{\mathrm{t}}}{k_{\mathrm{R}}}\right]^{2} \frac{S_{\mathrm{t}}(\theta)}{2 W_{\mathrm{i}}}
\end{aligned}
$$

où $W_{i}$ représente la puissance incidente normalisée donnée dans la référence [9] :

$$
W_{\mathrm{i}}=\frac{k_{\mathrm{t}}^{2} k_{\mathrm{R}}}{16 p}\left[\frac{1}{p^{2}}+\frac{2}{k_{\mathrm{R}}^{2}}+\frac{1}{s^{2}}-\frac{4}{k_{\mathrm{R}}(p s)^{1 / 2}}\right] .
$$

\section{Dispositif de mesure et procédure expérimentale.}

Nous avons étudié expérimentalement la conversion de mode par des rainures de forme rectangulaire usinées au centre de la face plane d'un demi-cylindre de duraluminium taillé dans un barreau de $200 \mathrm{~mm}$ de diamètre et de $40 \mathrm{~mm}$ de hauteur (Fig. $4 \mathrm{a}-4 \mathrm{~b}$ ). L Les dimensions de la rainure gravée suivant la direction de l'axe du cylindre sont respectivement de $3,28 \mathrm{~mm}$ et $4 \mathrm{~mm}$ pour la largeur ( $2 a$ ) et de $0,15 \mathrm{~mm}$ pour la profondeur $(h)$ (Fig. 4c). L'onde de surface est générée par la méthode du coin [11] et se propage jusqu'à la rainure sur une surface lisse. Le récepteur est solidaire d'un bras permettant de le faire tourner autour de la face courbe de l'échantillon.L'axe de rotation est confondu avec l'axe du demi-cylindre (Fig. 5). Ainsi, la distance entre l'émetteur et le récepteur reste constante au cours de l'expérience et fixée à $15 \mathrm{~cm}$. La position du récepteur est repérée à l'aide de l'angle $\theta$ défini comme l'angle entre le plan de l'interface air-échantillon et l'axe du récepteur. Cet angle peut varier entre $10^{\circ}$ et 

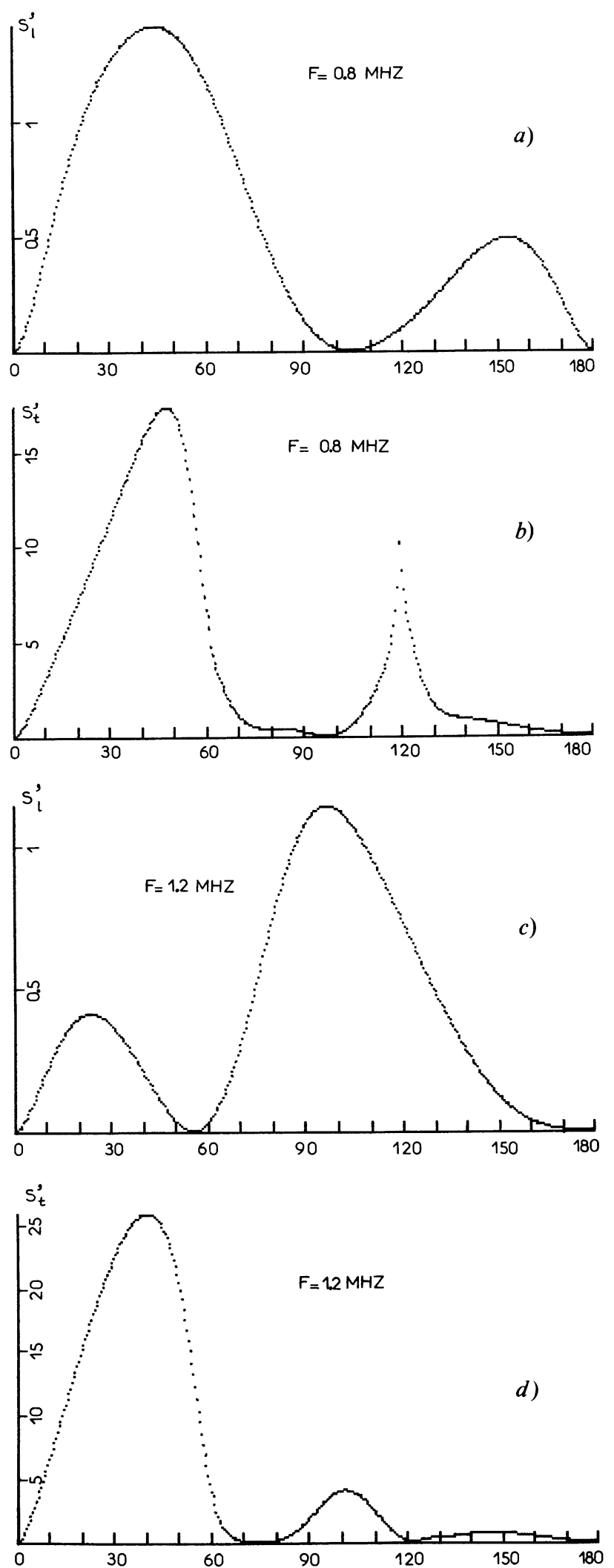

Fig. 3. - Courbes théoriques représentant les variations de $S_{1}^{\prime}$ et $S_{\mathrm{t}}^{\prime}$ en fonction de l'angle sagittal $\theta:$ a) $S_{1}^{\prime}(\theta) ; F=$ $0,8 \mathrm{MHz}$ b) $S_{\mathrm{t}}^{\prime}(\theta) ; F=0,8 \mathrm{MHz}$; c) $S_{1}^{\prime}(\theta) ; F=1,2 \mathrm{MHz}$; d) $S_{\mathrm{t}}^{\prime}(\theta) ; F=1,2 \mathrm{MHz}$.

[Theoretical curves of $S_{1}^{\prime}$ and $S_{\mathrm{t}}^{\prime}$ given by the Tuan's formulations : a) $S_{1}^{\prime}, F=0.8 \mathrm{MHz}$; b) $S_{1}^{\prime}, F=0.8 \mathrm{MHz}$; c) $S_{1}^{\prime}$, $F=1.2 \mathrm{MHz}$; d) $S_{\mathrm{t}}^{\prime}, F=1.2 \mathrm{MHz}$.]

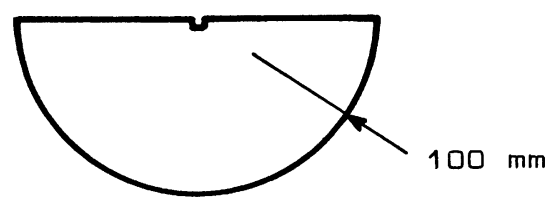

a)

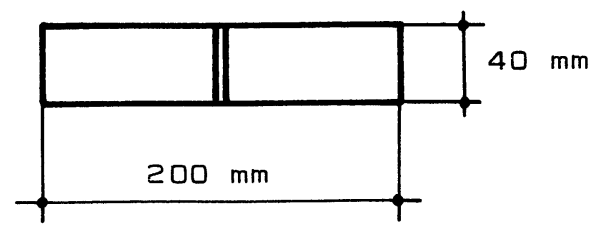

b)

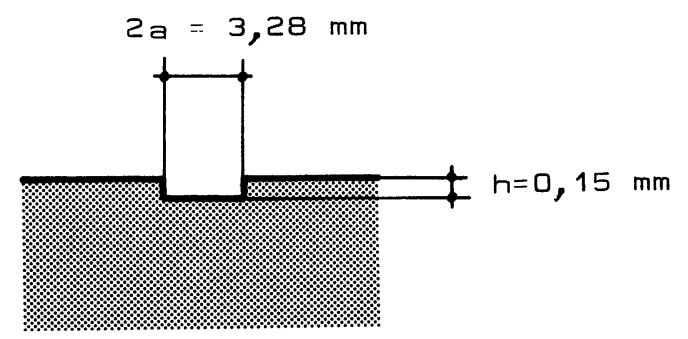

Fig. 4. - Description de l'échantillon.

[Description of the sample.]

$170^{\circ}$, compte tenu de l'encombrement des palpeurs. Le récepteur et l'émetteur sont disposés de telle façon que leur axe d'émission et de réception se situe dans le même plan contenant la normale à l'interface.

Le couplage entre le demi-cylindre et le récepteur est assuré par un fluide visqueux. Un système à vis permet d'appliquer une pression constante sur le récepteur de manière à assurer un couplage aussi reproductible que possible.

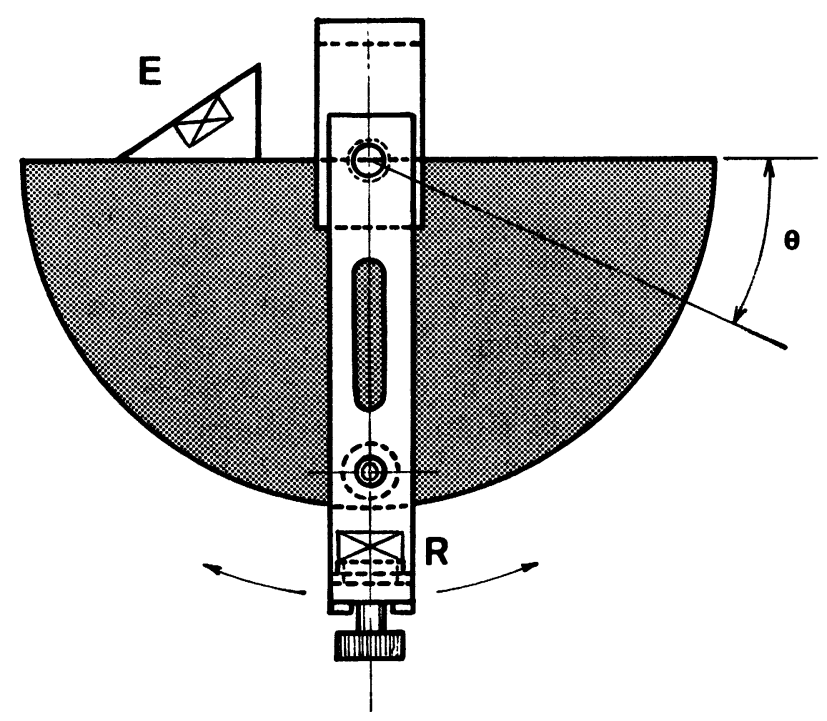

Fig. 5. - Dispositif mécanique.

[Mechanical arrangement.] 
L'émetteur est excité par des impulsions longues (6 $\mu \mathrm{s})$. Une chaîne classique de réception amplifie le signal reçu et le visualise sur l'écran d'un oscilloscope. Ce signal est le plus souvent constitué de plusieurs impulsions. Si l'identification de chacune d'elles s'avère difficile, il est relativement simple d'isoler le signal provenant de la conversion de l'onde de Rayleigh en onde de volume et diffusé dans la direction $\theta$. Les variations de l'amplitude de ce signal, en fonction de l'angle sagittal $\theta$, sont relevées tous les $5^{\circ}$ de $10^{\circ}$ à $170^{\circ}$. Suivant le type de l'onde de volume à détecter (longitudinale ou transversale), nous utilisons un palpeur sensible au déplacement particulaire longitudinal ou transversal. Les points expérimentaux correspondent à la moyenne des amplitudes effectuées sur trois relevés. Les fréquences de travail $(2,25$ et $1 \mathrm{MHz})$ sont telles que $h$ vérifie l'inégalité $h \ll \lambda_{\mathbf{R}}$.

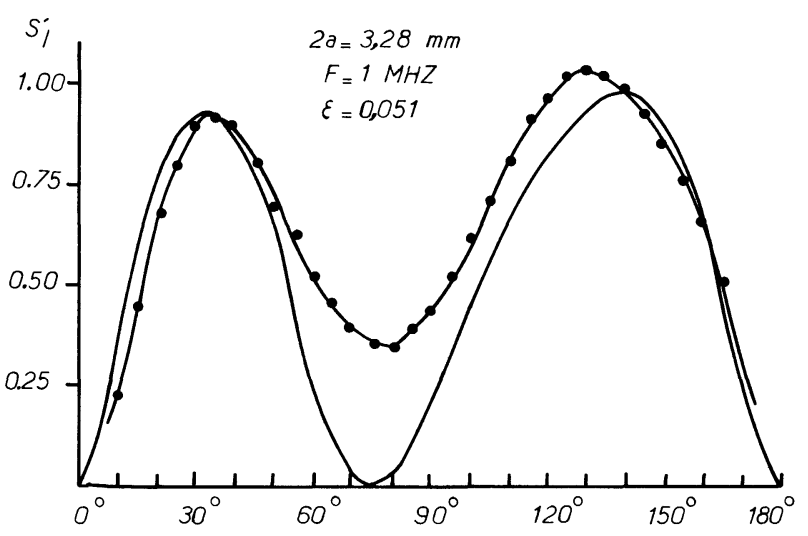

a)

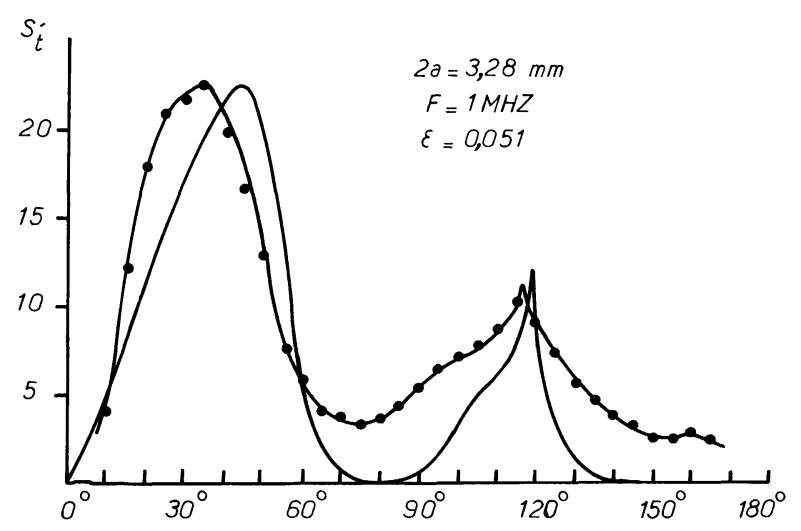

b)

Fig. 6. - Comparaisons des diagrammes angulaires théorique $S_{j}^{\prime}(\theta)(\longrightarrow)$ et expérimental $V_{j}(\theta)(-\cdot-)$. a) Cas de l'onde de volume longitudinale, $F=1 \mathrm{MHz}$ et $2 a=$ $3,28 \mathrm{~mm}$. b) Cas de l'onde de volume transversale, $F=$ $1 \mathrm{MHz}$ et $2 a=3,28 \mathrm{~mm}$.

[Comparison of the theoretical ( $\longrightarrow$ ) and experimental $(-\bullet)$ angular distributions of $S_{1}^{\prime}$-a) and $S_{\mathrm{t}}^{\prime}$-b) for $F=$ $1 \mathrm{MHz}$.]

REVUE DE PHYSIQUE APPLIQUÉE. - T. 20, № 6, JUIN 1985

\section{Résultats expérimentaux et discussion.}

La mesure de l'intensité incidente étant très difficile expérimentalement, nous n'avons pu que mesurer une quantité $V_{1}(\theta)$ [ou $V_{t}(\theta)$ ] proportionnelle à $S_{1}^{\prime}(\theta)$ [ou $\left.S_{t}^{\prime}(\theta)\right]$. Le coefficient de proportionnalité est essentiellement fonction des caractéristiques acoustiques du matériau dans lequel se propagent les ondes de volume engendrées au niveau de la rainure.

La largeur des rainures $(3,28 \mathrm{~mm}$ et $4 \mathrm{~mm})$ a été choisie de façon à ce que le coefficient de réflexion $R$ au niveau de la fente soit minimal [5] à la fréquence $F=2,25 \mathrm{MHz}$. Pour l'autre fréquence (1 MHz), la largeur de la fente $(3,28 \mathrm{~mm})$ correspond à une valeur intermédiaire de $R$.

Sur les figures 6 à 8 , nous avons représenté les variations angulaires (- - -) de $V_{1}(\theta)$ et de $V_{t}(\theta)$ observées

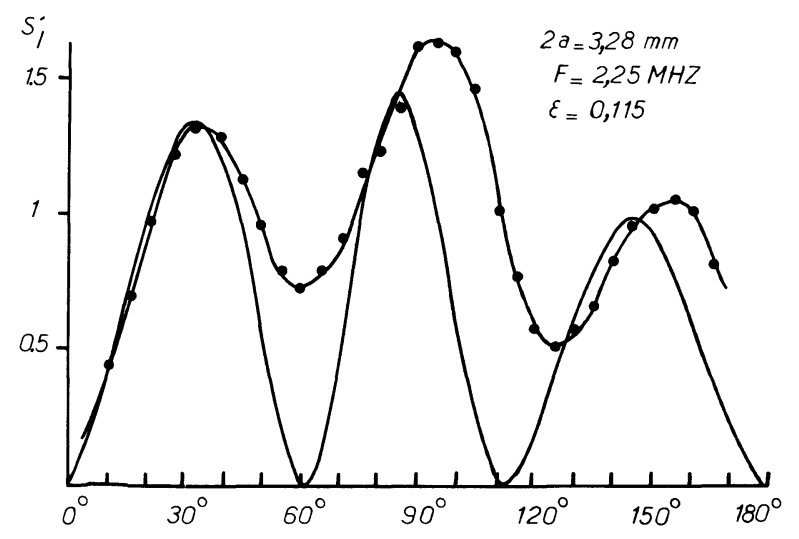

a)

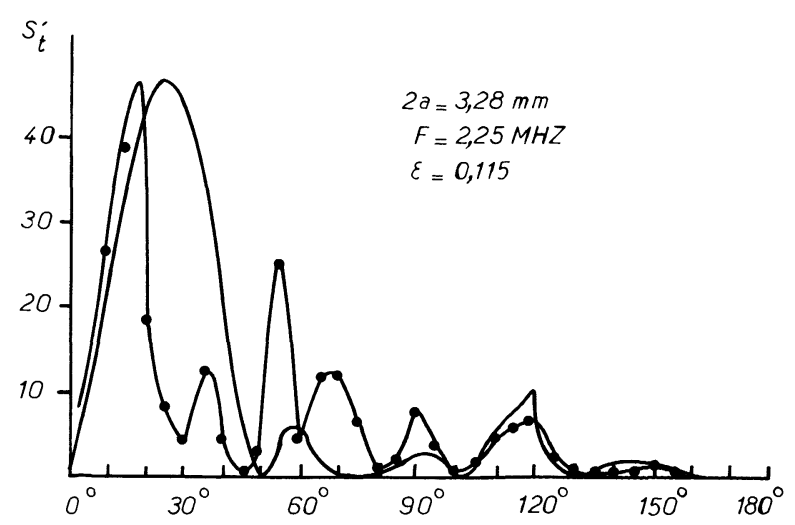

b)

Fig. 7. - Comparaisons des diagrammes angulaires théorique $S_{j}^{\prime}(\theta)(\longrightarrow)$ et expérimental $V_{j}(\theta)(-\bullet-)$. a) Cas de l'onde de volume longitudinale, $F=2,25 \mathrm{MHz}$ et $2 a=$ $3,28 \mathrm{~mm}$. b) Cas de l'onde de volume transversale, $F=$ $2,25 \mathrm{MHz}$ et $2 a=3,28 \mathrm{~mm}$.

[Comparison of the theoretical $(\longrightarrow$ ) and experimental $(-\bullet-)$ angular distributions of $S_{1}^{\prime}$-a) and $S_{1}^{\prime}$-b) for $F=$ 2.25 MHz.] 


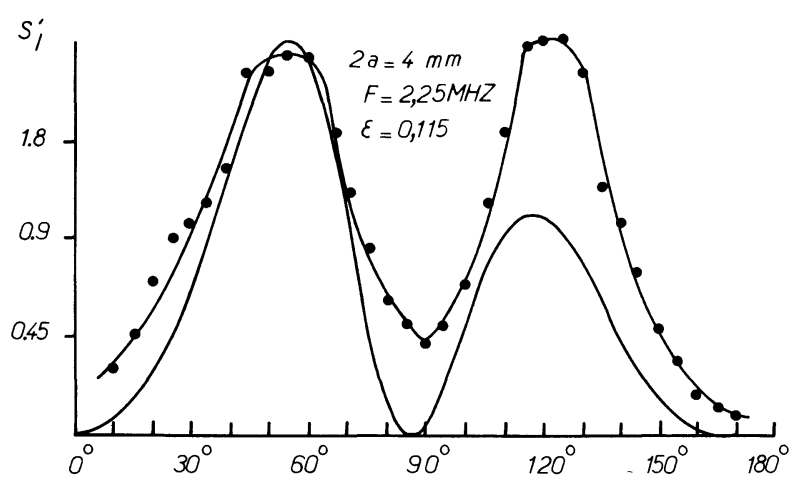

a)

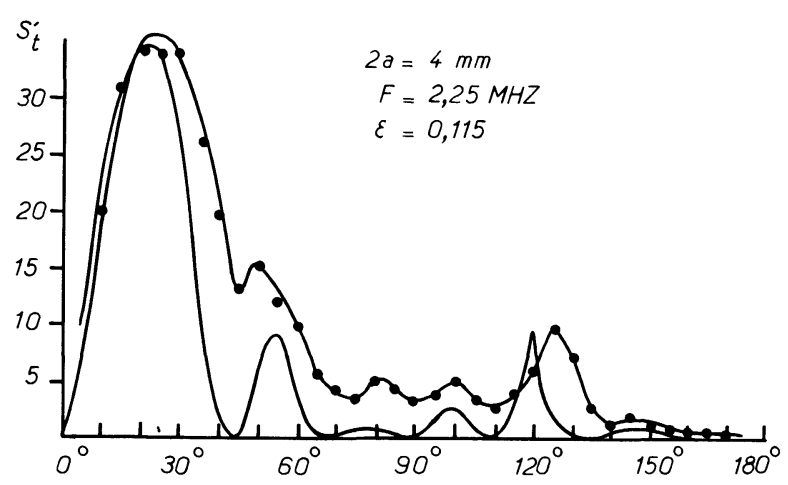

b)

Fig. 8. - Comparaisons des diagrammes angulaires théorique $S_{j}^{\prime}(\theta)(\longrightarrow)$ et expérimental $V_{j}(\theta)(-\bullet-)$. a) Cas de l'onde de volume longitudinale, $F=2,25 \mathrm{MHz}$ et $2 a=$ $4 \mathrm{~mm}$. b) Cas de l'onde de volume transversale, $F=$ $2,25 \mathrm{MHz}$ et $2 a=4 \mathrm{~mm}$.

[Comparison of the theoretical (-) and experimental $(\longrightarrow-)$ angular distributions of $\left.S_{1}^{\prime}-\mathrm{a}\right)$ and $\left.S_{t}^{\prime}-\mathrm{b}\right)$ for $F=$ $2.25 \mathrm{MHz}, 2 a=4 \mathrm{~mm}$.]

expérimentalement pour différentes largeurs du profil ( $2 a=3,28$ et $4 \mathrm{~mm})$ et diverses fréquences $(F=1 \mathrm{MHz}$ et $2,25 \mathrm{MHz}$ ). Ces courbes expérimentales sont arbitrairement normalisées par rapport au premier maximum observé sur la courbe théorique (-) tracée à titre de comparaison pour chaque expérience et représentant les variations angulaires de $S_{1}^{\prime}(\theta)$ ou $S_{t}^{\prime}(\theta)$.

On observe entre les deux tracés (théorique et expérimental) un assez bon accord : les positions théoriques des maxima et des minima sont confirmées par l'expérience; l'écart est inférieur à $10^{\circ}$. A l'exception des courbes représentées sur les figures $7 \mathrm{~b}$ et $8 \mathrm{a}$, les variations angulaires $V_{1}(\theta)$ et $V_{t}(\theta)$ respectent l'amplitude relative des pics entre eux et les minima sont peu prononcés à cause de l'ouverture des palpeurs utilisés en réception.

Un effet caractéristique domine les variations de $V_{1}(\theta)$, c'est la présence pour des angles d'incidence $\theta>70^{\circ}$, d'un " glissement " progressif de la courbe par rapport aux variations théoriques $S_{1}^{\prime}(\theta)$. Ce «glisse- ment » est particulièrement visible sur la figure $7 \mathrm{a}$; il ne peut s'expliquer par une imprécision sur la valeur des dimensions de la fente qui sont définies à $10^{-2}$ près. En revanche, une analyse théorique systématique de l'influence de la fréquence sur le diagramme de diffusion a montré qu'une variation de la fréquence de l'onde incidente de $5 \%$ entraîne un "glissement progressif » de la courbe $S_{1}^{\prime}(\theta)$ pouvant atteindre $10^{\circ}$ pour les valeurs élevées de $\theta$. Une modification de la fréquence de résonance au cours de l'expérience peut donc rendre compte de ces désaccords. Ce changement de fréquence peut être aussi le résultat de l'interaction de l'onde de surface avec la rainure comme cela a été prévu théoriquement par Maradudin [12] dans le cas d'une surface rugueuse aléatoire.

En ce qui concerne les tracés de $V_{t}(\theta)$, on note un bon accord entre les valeurs expérimentales et les prévisions théoriques pour les valeurs de $\theta>70^{\circ}$ dans le cas de la rainure de largeur $2 a=3,28 \mathrm{~mm}$. Pour l'échantillon possédant la rainure de largeur $2 a=4 \mathrm{~mm}$, l'accord est bon quel que soit l'angle d'incidence. Aux restrictions angulaires près mentionnées ci-dessus, la position des pics et des minimum est assez bien respectée de même que l'amplitude relative des pics entre eux.

Dans le cas où $\theta<70^{\circ}$, les désaccords observés se manifestent par le déplacement du premier pic (cf. Fig. 6b) ou par la présence d'oscillations qui traduisent l'existence d'interférences dans cette zone angulaire particulière (cf. Fig. 7b). Nous avons attribué l'origine de ces différences (entre la théorie et l'expérience) aux effets liés à l'interface solide-solide [13,14] et à la façon dont est créée l'onde de surface.

Du fait de l'ouverture du faisceau dans le coin en contact avec l'échantillon, des ondes de volume sont générées dans les deux milieux en plus de l'onde de surface. C'est ainsi que la présence d'une onde « transversale directe " a été mise en évidence pour des valeurs de $\theta$ inférieures à $40^{\circ}$. Ce faisceau dont la structure dépend de la forme de l'émetteur (rectangulaire ou circulaire) peut interagir avec l'onde de volume transversale engendrée lors de la conversion de surface en onde de volume et donne naissance à des interférences. Rappelons enfin que si une onde incidente transversale à polarisation horizontale $(\mathrm{SH})$ est utilisée pour générer l'onde de Rayleigh, il n'existe qu'une seule onde transmise et réfléchie, ce qui devrait avoir pour effet de diminuer les effets parasites. Ce résultat semble être confirmé par la courbe représentée sur la figure $8 \mathrm{~b}$. Au cours de cette expérience, l'onde de surface était générée à l'aide d'une onde incidente $\mathrm{SH}$, contrairement aux autres expériences effectuées sur l'échantillon de largeur $3,28 \mathrm{~mm}$ pour lesquelles une onde à polarisation longitudinale $(\mathrm{P})$ a été utilisée.

\section{Conclusion.}

Les mesures expérimentales que nous avons obtenues donnant la répartition directionnelle de l'intensité transportée par les ondes de volume confirment la 
validité du modèle théorique développé par Parekh et Tuan dans le cas où $h / \lambda_{\mathrm{R}} \ll 1$, et soulignent la très grande influence des conditions expérimentales pour ce type d'expérience.
Une analyse critique des courbes expérimentales jointes à une étude des conditions de réalisation de l'expérience montre que l'utilisation de palpeur à onde SH et circulaire est bien adaptée à ce genre d'étude.

\section{Bibliographie}

[1] Ronnekleiv, A., Souquet, J. et Shaw, H. J., Grating acoustic scanners, Ultra. Symp. Proc. IEEE cat 75, p. 91-93.

[2] TuAN, H. S. et LI, R. C. M., Rayleigh wave reflection from groove and step discontinuities, J. Acoust. Soc. Am. 55 (1974) 1212-1217.

[3] Simons, D. A., Reflection of Rayleigh waves by strips, grooves and periodic arrays of strips or grooves. J. Acoust. Soc. Am. 63 (1978) 1292-1301.

[4] Tuan, H. S., On bulk waves excited at a groove by Rayleigh waves. J. Appl. Phys. 46 (1975) 36-41.

[5] Parekh, J. P. et Tuan, H. S., Reflection and bulk wave conversion of Rayleigh wave at a single shallow groove. J. Appl. Phys. 48 (1977) 994-1003.

[6] Chang, C. P. et Tuan, H. S., On the surface-to-bulk mode conversion of Rayleigh waves. IEEE Trans. Microwave Theory Tech. MTT 21 (1973) 558-560.

[7] Tuan, H. S. et Chang, C. P., Tapping of Love waves in an isotropic surface wave guide by surface-to-bulk wave transduction. IEEE Trans. Microwave Theory Tech. MTT 20 (1972) 472-477.
[8] MoRse, P. M. et FeshbaCK, H., Methods of Theoretical Physics, vol. 2 (New York, Mc Graw-Hill) 1953, chap. 9.

[9] Ronnekleiv, A. et Souquet, J., On the theory of acoustic surface-wave to bulk wave scattering by groove. J. Appl. Phys. 47 (1976) 4422-4425.

[10] RonNekleiv, A. et SouQueT, J., Surface wave to bulk wave scattering from grooves. Ultra. Symp. Proceedings IEEE cat 75, p. 434-437.

[11] Vixtorov, I. A., Rayleigh and Lamb waves. Physical theory and Applications (Plenum Press, New York) 1967.

[12] Maradudin, A. A. et Mills, D. L., Attenuation of Rayleigh surface waves by surface roughness. Ann. Phys. 100 (1976) 262-309.

[13] Bertoni, H. L. et TAMIR, T., Characteristics of wedge transducers for acoustic surface waves. IEEE Trans. Ultra. SU-22 (1975) 415-420.

[14] Bertoni, H. L., Coupling layers for efficient wedge transducers. SU-22 (1975) 421-430. 